\title{
Knowledge based Grid Using Semantic Web for the University System
}

\author{
Muqeem Ahmed \\ Deprtment of computer science \\ Jamia Millia Islamia \\ New Delhi India
}

\author{
S.Zeeshan Hussain \\ Deprtment of computer science \\ Jamia Millia Islamia \\ New Delhi India
}

\author{
S.A.M. Rizvi \\ Deprtment of computer science \\ Jamia Millia Islamia \\ New Delhi India
}

\begin{abstract}
The emergence of semantic web will result in an enormous amount of knowledge base resources on the web. The efficient use of knowledge resource totally depends on internet search engines which do not only help organization who are involved in the development of web browsers but also international economy as a whole buy facilitating efficient transition, managing and sending appropriate results to the web browser user. It is observed that data flowing from internet is often vague and therefore there is need to develop technology which has to get relevant and focused information. The Knowledge base grid in semantic web is an important instrument to enhance the application domain of semantic web. using the semantic web technologies for the solution of the problems of the students in the knowledge based grid distributed environment is major challenging task where they can collect coordinate publish and share knowledge to get the the admission in the course according to their ability. they can get knowledge about different universities, colleges and institutions in the distributed environment within these they select different courses according to their ability for decision making for their career and also find jobs available in the different colleges, universities and institutions in the distributed environment.
\end{abstract}

\section{Keywords}

Grid Computing, semantic web. Distributed Environment and ontology.

\section{INTRODUCTION}

Grid was introduced in the mid1990s to denote a distributed computing environment for advanced science and technology. The concept of Computational Grid has been inspired by the 'electric power Grid', in which a user could obtain electric power from any power station present on the electric Grid irrespective of its location, in an easy and reliable manner. When we need additional electricity we have to just plug into a power Grid to get additional electricity on demand, similarly for computational resources plug into a Computational Grid to access additional computing power on demand using resource. Internet users require lots of knowledge for their future need but problem is irrelevancy of data. The present web and search engines are fully based on syntax to retrieve the data from the distributed environment which does not full fill internet user requirement. the scalable repository permits the fusion and reuse of knowledge from various resources. In an operational context these knowledge base features need to be combined with more traditional information retrieval ideas that gives both cross gained precision based knowledge retrieval [1]. the Semantic web play important role to retrieve information on the basis of meaning which is an extension of the current web in which information is given well-defined meaning, better enabling computers and people to work in cooperation (Tim Berners-Lee et al.)[2].But the information overloading was the major challenging research issue. Fran Berman came with new idea and provides the concept of Knowledge Grid as the convergence of a comprehensive computational infrastructure along with the scientific data collections and applications for routinely supporting the synthesis of knowledge from that data [3].Various researchers used the knowledge based grid for knowledge sharing and knowledge managing globally in the distributed environment considered knowledge in three dimensional knowledge space and provides operation language to create knowledge grid, put, edit knowledge to open grid as required [4].Zhuge believed that "Knowledge Grid is an intelligent and sustainable Internet application environment that enables people or virtual roles (mechanisms that facilitate interoperation among users, applications, and resources) to effectively capture, publish, share and manage explicit knowledge resources. It also gives on-demand services to support innovation, cooperative teamwork, problem-solving and decision making. It incorporates epistemology and ontology to reflect human cognition characteristics; exploits social, ecological and economic principles; and adopts the techniques and standards developed during work toward the next-generation web." [5] In addition, he find out that " Knowledge Grid Environment consists of autonomous individuals, self-organized semantic communities, an adaptive networking mechanism, an evolving semantic overlay keeping meaningful connection between individuals, flows for dynamic resource sharing, and mechanisms supporting effective resource management and providing appropriate knowledge services for problem-solving and innovation. It supports innovation and harmonious development of science, technology and culture"[6]. The knowledge based workflow exploited as the platform for knowledge sharing and knowledge service to provide the functions of knowledge discovery that will be very effective for the electronic commerce system in the distributed environment [7] to synthesizes the integration of the data, computing, and the network hardware, the development of the software, and the collaborations of a large and distributed infrastructure. The ontology plays the very effective role for the semantic knowledge creation and representation in the knowledge grid for knowledge discovery in distributed environment system by using some data mining tools, techniques and algorithm as the development methods in the distributed grid environment knowledge will be refined which will be very effective for student information management related applications [8]. It offer the effective and efficient support to the process of organizational and educational knowledge discovery on the 
basis of agent technology and integrate grid data resource which further improve services of the users of the university like searching, advisory and help desk system, course and resource management, student and faculty communication etc in the distributed environment[9]. From the view of above literature we understand that the Knowledge Grid is a knowledge carrier and good controlled knowledge collections, and it defines the operation mode of Knowledge Management in distributed environment. It can be developed on the semantic web which is the integration of semantic web and grid and provides people semantic browse by taking many knowledge bases as its knowledge sources. Lots of work has been done on the application of the knowledge based grid in semantic web but the very little amount of work has been done in the area of university domain which will be very help full for the student for the decision making. this paper consider all the developments issues of knowledge based grid in semantic web for the university domain where student can search ,collect ,coordinate, publish and share knowledge in the distributed environment. Four important component identified and developed for the development of knowledge based grid in semantic web semantic interface for retrieving semantic related information, knowledge server acting as a web container for knowledge ,ontology server for managing ontology and directory knowledge server acting as a catalog of knowledge base.

\section{KNOWLEDGE BASED GRID}

The knowledge grid consider as a mechanism that covers a for uniformly storing knowledge as a knowledge space and for sharing and managing knowledge operation interface, the knowledge in space [10]. It established on either the semantic web or the high-performance grid computing environment, that enables users or virtual rolls to conveniently publish, acquire, share, and manage knowledge resource or supply knowledge service to demanders and help them in cooperative work, problem solving, decision support, and knowledge innovation. The architecture of the knowledge grid consists of two layers core knowledge grid layer and high level knowledge grid layer. Over the knowledge grid the former layer refers to knowledge services directly implemented on the top of generic grid services, the latter is used to describe, develop and execute knowledge discovery computations.

The architecture of knowledge grid is illustrated in Figure 1.

Fig 1: Architecture of the Knowledge Based Grid

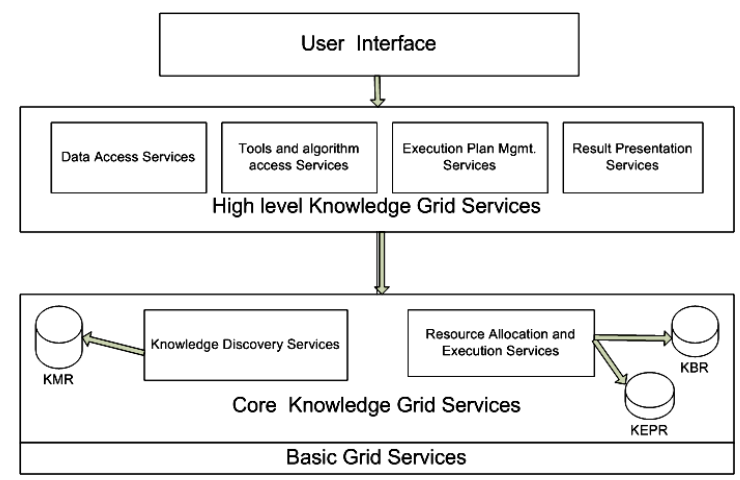

The knowledge grid consists of all sorts of knowledge servers: Ontology server (store basic ontology knowledge: concepts, instances, attributes, and their relationships), Case server (store design knowledge, design experience, design patents, design cases, etc.), Rule server (store the design rules, innovation principles, TRIZ contradiction matrix, etc.), Knowledge Map server (supply knowledge index service, including knowledge notes register, semantic register), and Workflow server (store workflow models in enterprises).

The knowledge grid layer, the knowledge supply layer is constructed for more efficient and agile applications of knowledge in the process of innovative design which consists of: knowledge query for problem solving, knowledge proactive recommendation, knowledge publishing, knowledge evaluation, and knowledge charge involving in the knowledge exchange. Those knowledge servers (ontology servers, knowledge map servers, cased servers, rules servers, and workflow servers) among one sub grid of enterprises are connected to form a virtual organization, and some sub grids of enterprises that belong to the same industry may constitute a knowledge union, which will promote knowledge sharing and exchange among enterprises in the same industry. The Knowledge Based grid refined the data by using the Knowledge Based mining and serves the refine web pages to the appropriate search. The Semantic interface sends a request to the Internet and that it links to different knowledge based components and finally with the usage of ontology [11] it is able to give to refined results. The mapping can be clearly defined in the figure below.

\section{Components of the Knowledge based grid using semantic web for the university system}
A. Semantic interface
B. Ontology server for managing ontology
C. Knowledge server as a web container for the knowledge
D. Knowledge Directory Server

The knowledge service of university KB grid

a. University $\mathrm{KB}$ ontology services

b. University KB grid inference services

- $\quad$ Forward chaining inference service

- $\quad$ Backward chaining inference service 


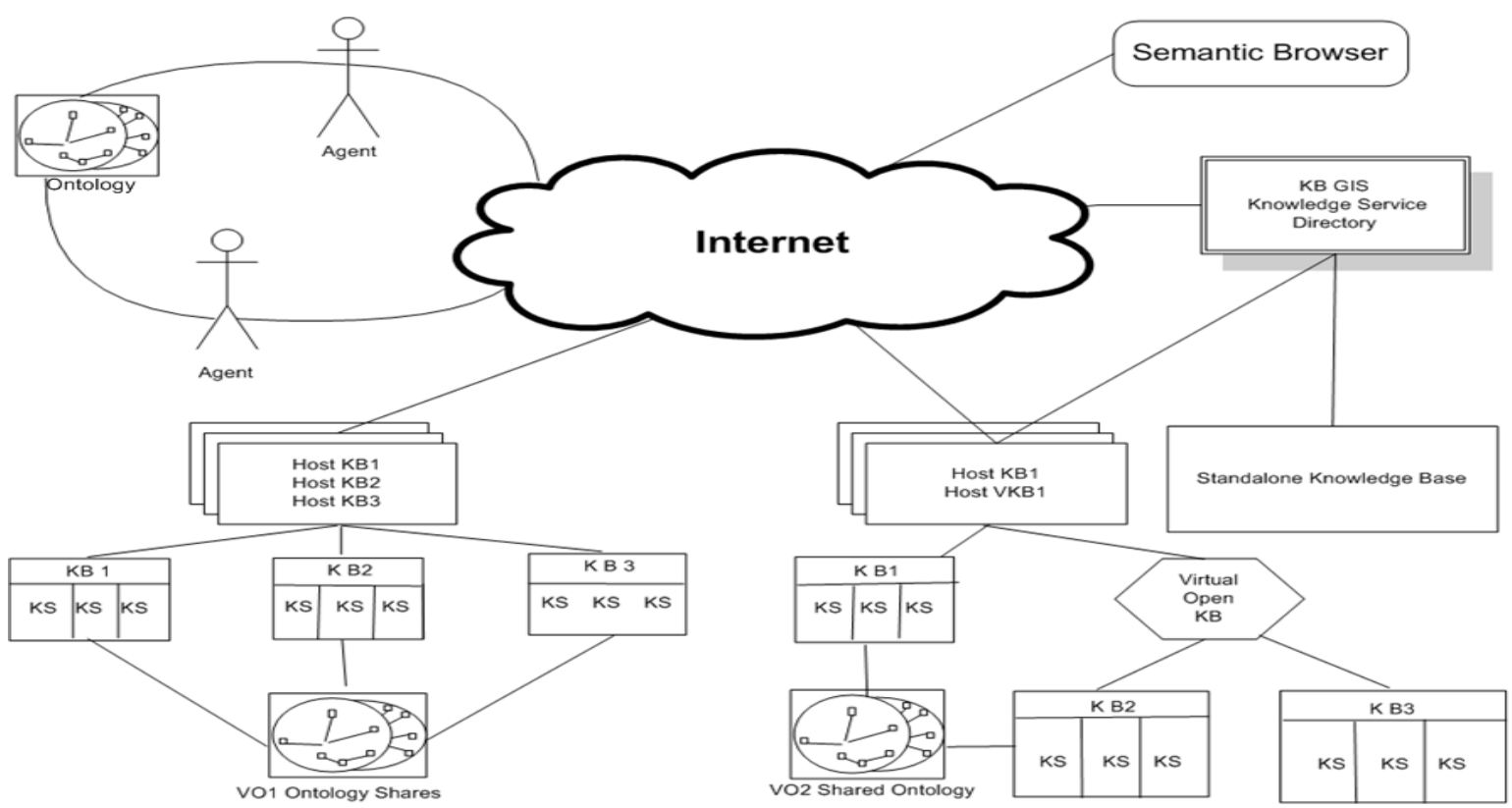

Fig 2: Framework of the knowledge Based grid using semantic web for the university

\section{THE WORKING SCENARIO OF UNIVERSITY BASED KNOWLEDGE GRID IN SEMANTIC WEB}

It is a process through student tries to find out the proper course through the university knowledge base grid

\section{Ontology browser}

Suppose someone called a want to take admission in a university. He does not know the course will be suitable for his. As we mentioned above he get the ontology graph by semantic browser during the semantic browsing processing A can find that node "course" has the property course "criteria "for admission at this time A only get the university ontology without any instances therefore course and criteria now have no real corresponding information

2. Course search $\mathrm{A}$ input the detail criteria minimum percentage semantic browser goes to the course $\mathrm{KB}$ to find out and display the course which have the same course as A inputs in the course $\mathrm{KB}$, criteria are stored as the course property, at the starting A may see many course that have the criteria of minimum percentage of qualifying exam.A can input more criteria to diminish the range of the courses till the A confirm what course he gets. During this process the semantic browse service and correlative query service work all the time

\section{University finding}

When he knows course name like MCRC a wants university $\mathrm{KB}$ grid to tell him which university is useful for him. Still by the semantic browser he find the node MCRC in the university ontology however unfortunately in this node property list there is not university that is to say in the course $\mathrm{KB}$ there are not any nodes to show which university has virtue for this course and A does not know what course is suitable for his /her.

\section{Correlative Course search}

One feasible way is to find out the same kind of course of MCRC which has almost the same attribute and then refer to their university now subsumption service start to work in the course KB, KB grid finds out all the courses which have same attributes as the MCRC .semantic browser interface list them with all their property to help. Student selects the most relevant.

\section{Correlative criteria finding}

Suppose advanced diploma in MCRC is selected from the course $\mathrm{KB}$, the university $\mathrm{KB}$ grid knows the minimum criteria. then the university $\mathrm{KB}$ grid goes to course $\mathrm{KB}$ to get the information about the advanced diploma in MCRC if advanced diploma in MCRC has some attribute of MCRC. Then university grid gives the advice that the student $\mathrm{KB}$ should take the admission in MCRC.

\section{IMPLEMENTATION OF THE KNOWLEDGE BASED GRID}

\subsection{Creation of knowledge base}

The Ontology has been created by using the different resources of computing in the Grid environment using the relations properties concept of these resources has been defined properly. so that the characteristics of any resource can be defined by its properties. The grid node accesses the resource description module and retrieves the information of the resource by executing ldap queries on those nodes and updates into the ontology. every type of information retrieved from the Grid node, the module creates an instance of the appropriate concept in the ontology forming conformity relation between instances and their respective types of concept. The instance will be exactly related to only the type of concept. Moreover, the values of various properties retrieved from MDS are assigned to the respective properties of the appropriate concepts in the ontology. The ontology with concepts and properties and the corresponding instances and property values together constitute the knowledge base of the 
Grid resources at this point. These types of the knowledge base can be created using the protégé-OWL libraries which contains the necessary and sufficient APIs for the creation of the instances for a concept, assign different values to properties and other ontology operations. This type of semantic description of resources facilitates the use of inference engines to interact with the knowledge base and retrieves information semantically in the distributed environment. Moreover, the description module is made to execute periodically so that the removal and addition of the different resources is updated in the knowledge base dynamically. The java implemented Algernon packages are used to query the ontology knowledge base. The package offers several java APIs with which various queries can be executed.

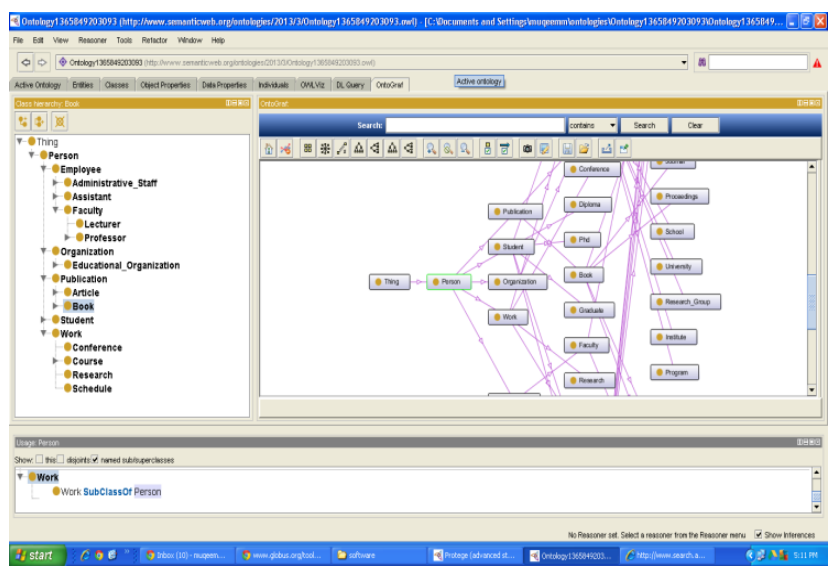

Fig 3: University ontology Meta data for the knowledge based grid

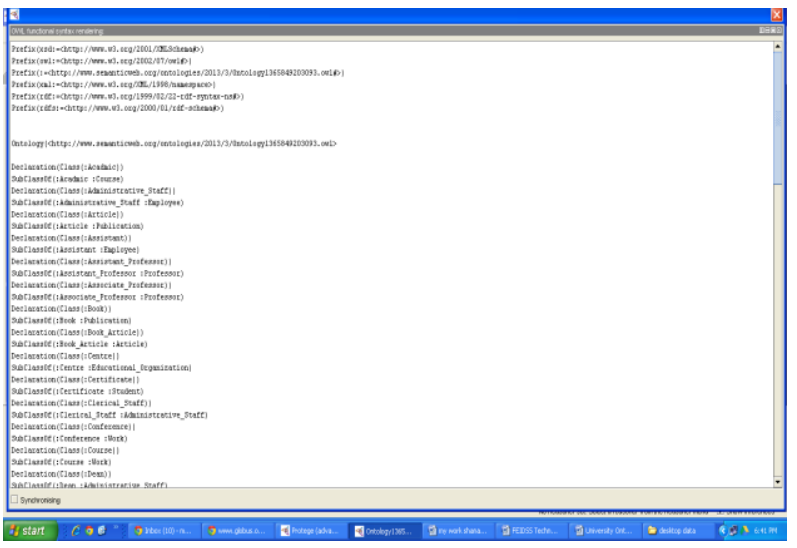

Fig 4:

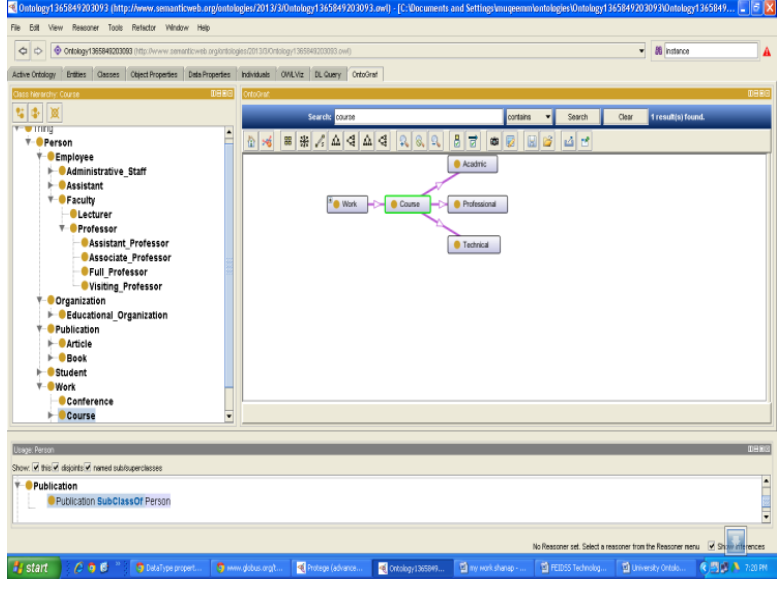

Fig 5:

\subsection{Comparision of constructed ontology with the reference ontology using Golden method}

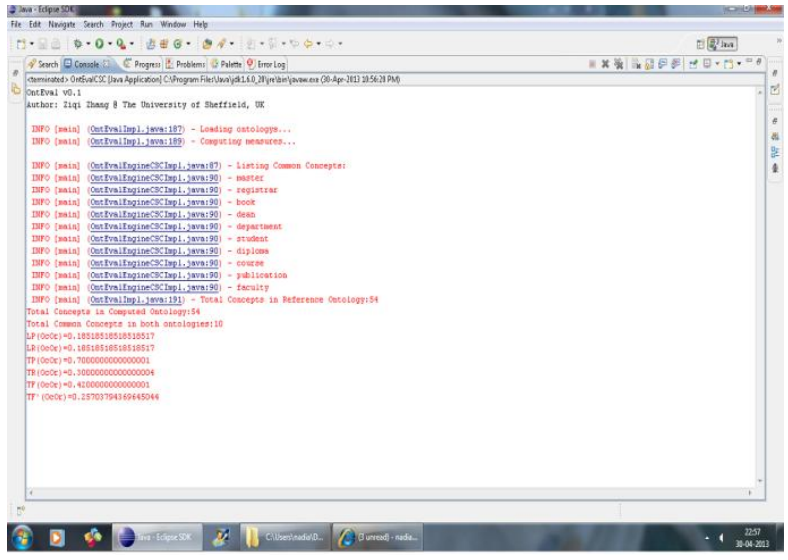

Fig 6: Compression Results of Ontology 


\subsection{Semantic establishment for the knowledge based grid in semantic web}

INPUT : A simple semantic (concept or property) from the 'semantic requirement' (S)

OUTPUT: the concepts which are either directly or indirectly related to the input concept/property

(Result).

\section{BEGIN}

1. Result $=$ NULL;

2. if ( $\mathrm{S}$ is Concept )

\{

if ( $\mathrm{S}$ is Primitive )

Result $=$ Result U S;

else if ( $\mathrm{U}$ is union or $\mathrm{S}$ is intersection )

Loop through each Concept in S

$\mathrm{C}=\operatorname{next}(\mathrm{S})$

Result $=$ Result U C;

3. else if ( $\mathrm{S}$ is Property )

Concept domain $=$ domain $(S)$;

do step 2 for Conceptdomain;

Concept range $=\operatorname{range}(\mathrm{S})$;

do step 2 for Concept range ;

\}

4. temp $=$ NULL;

5. Loop through each Concept in Result

\{

$\mathrm{C}=\operatorname{next}($ Result $)$;

temp = temp $\mathrm{U}$ getEquivalentConcepts $(\mathrm{C})$;

\}

6. Result $=$ temp U Result

7. Return Result;

END

\subsection{Accessing the Protégé knowledge Base}

We have used a rule-based inference engine in Java to open a Protégé knowledge base and use Algernon to query the KB. it, will be run to change the path in UNIVERSITY_PROJECT to match the correct project on the system.

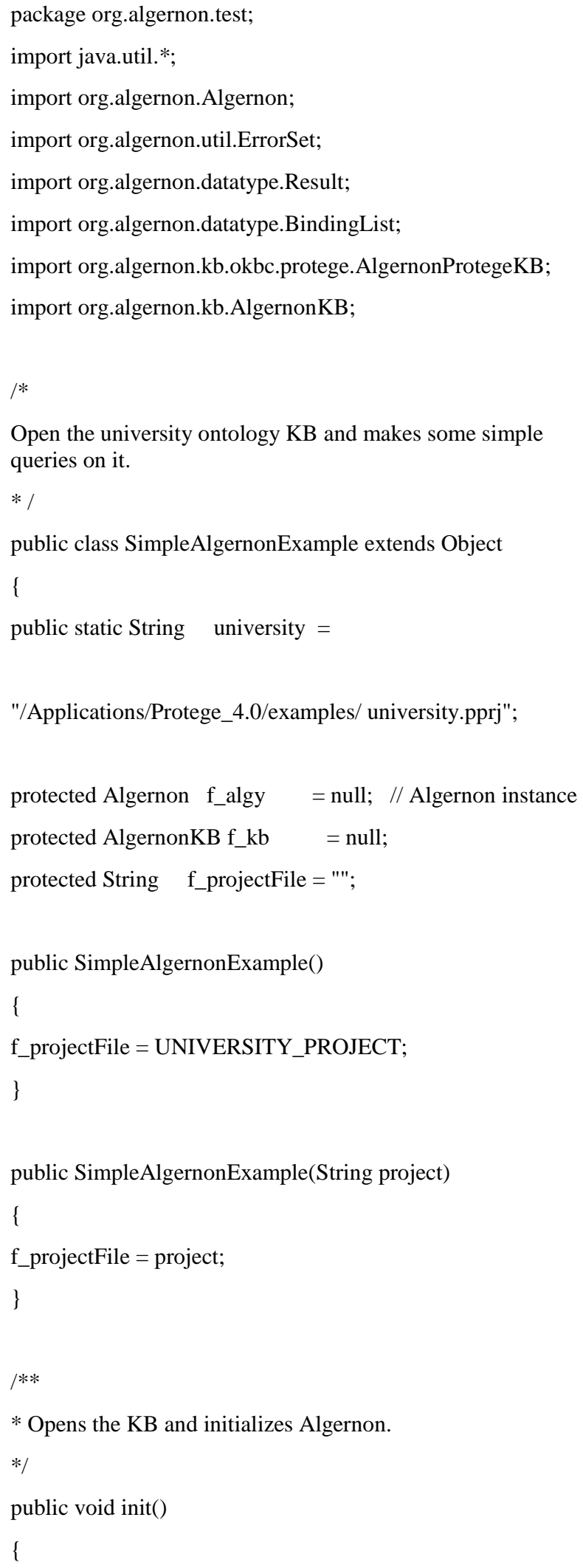




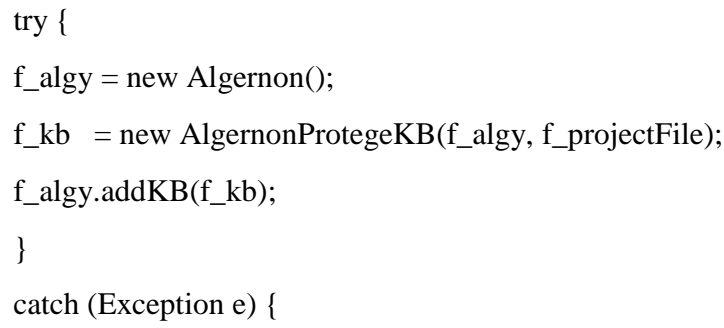

// One way to access the results is to iterate on the results // using result.iterator(). Each element of the iterator

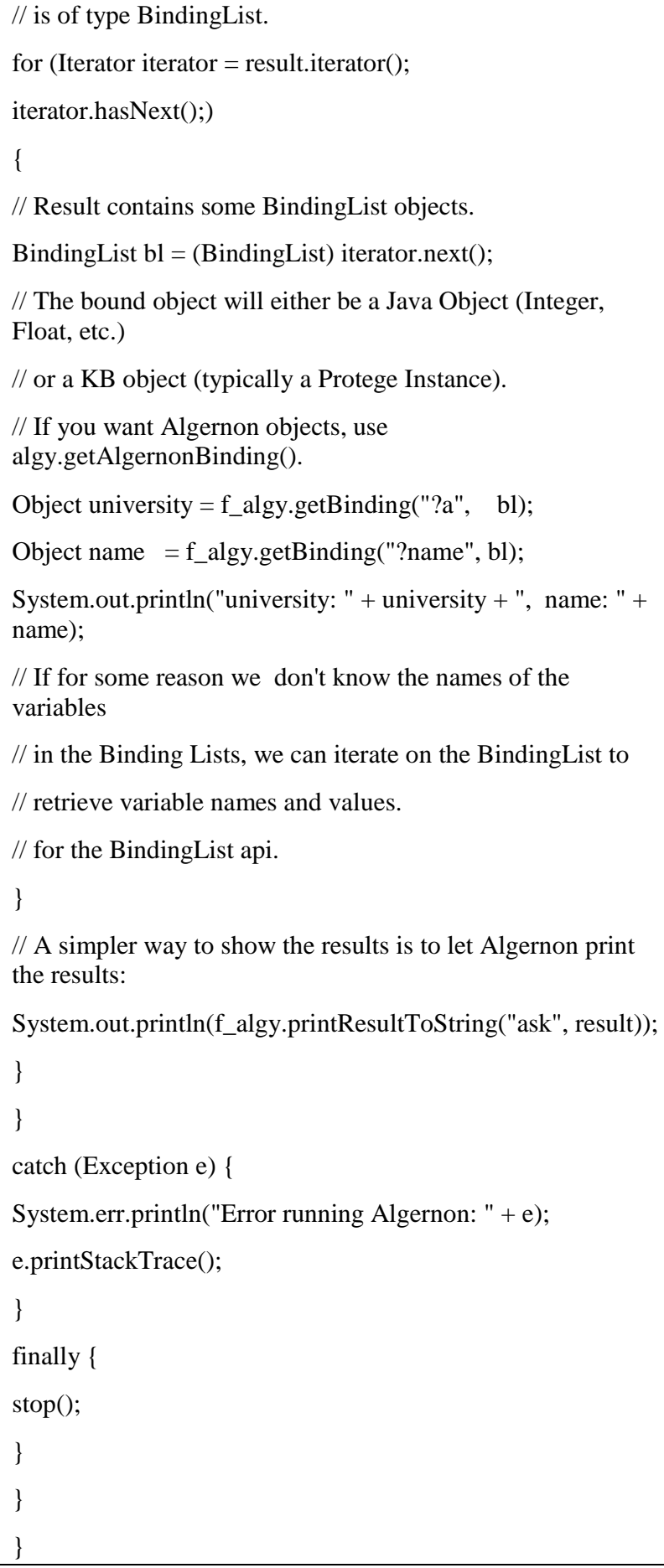

// If for some reason we don't know the names of the variables

// in the Binding Lists, we can iterate on the BindingList to // retrieve variable names and values.

// for the BindingList api. \}

// A simpler way to show the results is to let Algernon print the results:

System.out.println(f_algy.printResultToString("ask", result)); \} \} catch (Exception e) \{ System.err.println("Error running Algernon: " + e); e.printStackTrace();

\}

finally \{

stop();

\}

\}

\}

\section{RESULTS AND DISCUSSION}

The resource discovery performance of the proposed semantic-based knowledge retrieval has been evaluated by comparing the amount of results obtained from 1 keywordbased searching mechanisms by the different resource requirement requests which were converted into the different 'queries' describe above . Across the Grid resources Semantic description is made to run periodically that contact the MDS, aggregate resource knowledge, and create knowledge base. Queries were executed through conventional matchmaking mechanism without considering the semantic present in the request initially. And for the resource discovery over the 
knowledge base the requested queries were all converted into Algernon query.

It has been concluded that the semantic-based searching mechanism retrieved more closely matching based on the experimental results, resources and thus resulted in greater than the conventional searching mechanism in the figure 7 . This is because, the user is not exactly match with available resource described in the knowledge base by the requested resources. The semantic component is responsible to retrieve resources that exhibit subsumption relationships with the request resources.

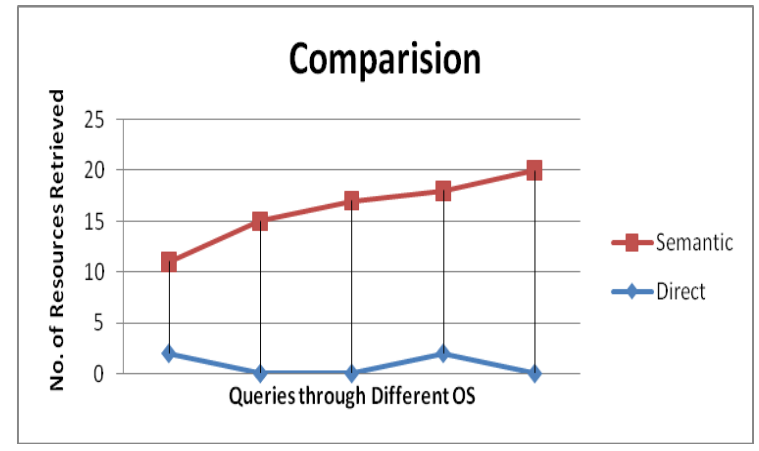

Fig 7:Direct VS Semantic Result for the

\section{CONCLUSION}

The efficient use of knowledge resource totally depends on internet search engines that do not only help organization who are involved in the development of web browsers but also international economy as a whole buy facilitating efficient transition, managing and sending appropriate results to the web browser user. It is observed that data flowing from internet is often vague and therefore there is need to develop technology which has to get relevant and focused information. The Knowledge base grid in semantic web which is an important instrument to enhance the application domain of semantic web. The knowledge grid based knowledge environment in the university domain using the semantic web for the solution of the problems of the students in the distributed environment where they can collect coordinate publish and share knowledge to get the admission in the course according to their ability which is the one of the major challenge for the improvement of the technology by providing on demand robust services in the distributed environment . In future the knowledge based grid system will improve the web application by the modification of the ontology knowledge base to solve the problem of internal security of the country by communicating the more nodes with the knowledge based grid that is the one of major research and development issue now days.

\section{REFERENCES}

[1] Martin P and peter"knowledge retrieval and world wide web" W Eklund Griffith university 2000

[2] Berners T., J. Hendler, and O. Lassila. The Semantic Web. Scientific American, May 2001.

[3] Berman F, "From teragrid to knowledge grid", Communication of the ACM, Volume 44, Issue 11, Nov. 2001, pp. 27 - 28.

[4] Zhuge H. ,"knowledge grid model and platform for global knowledge sharing" expert system with applications 22 (2002)313-320

[5] Zhuge H world scientific co pt ltd ISBN 981-256-140-4 2004

[6] Zhuge H , China's e-Science Knowledge Grid Environment, IEEE Intelligent Systems, Volume 19, Issue 1, Jan-Feb 2004, pp. 13 - 17 , doi: 10.1109/MIS.2004.126587919.

[7] Ahmed M."knowledge grid "Knowledge Grid Electronic Commerce Recommendation System in the Distributed Collaborative Framework" International Journal of Computing Technology and Information Security Vol.1, No.2, pp.1-8, December, 2011. ISSN: 2231-1998 () 2011. www.ijctis.com.

[8] Ahmed et al. "Ontology Based Knowledge Grid in Semantic Web to Discover Knowledge in Distributed Environment", Orient. J. Comp. Sci. \& Technol., Vol. 4(2), 261-272 (2011)

[9] Ahmed M.” Knowledge grid based knowledge discovery in distributed environment "978-1-4244-7818-7/10/ c_2010 IEEE

[10] Zhen L , Jiang Z, Liang J" Knowledge grid-based problem-solving platform” Int J Adv Manuf Technol (2009) 42:1217-1229

[10]Bruce,Geoffrey,YozhongSemanticGridPrimer http://www.semanticgrid.org/GGF/ggf9/discussion.ppt)

[11] HO Tu Bao, INTRODUCTION TO KNOWLEDGE DISCOVERY AND DATAMINING, Institute of InformationTechnology http://www.netnam.vn/unescocourse/knowlegde/1.1.htm)

[12] David J. Skyrme, Knowledge Networking Creating the Collaborative Enterprise, 1999 page 42. 